\title{
Cambio de actitud y clima del aula mediante el uso de metodología cooperativa en materias de bajo interés para el alumno
}

\author{
María Pilar Flores Asenjo \\ Universidad Católica San Antonio de Murcia \\ pflores@ucam.edu
}

Recibido: 09/10/2012

Aceptado: 23/01/2013

\begin{abstract}
Resumen
Partiendo de la estrecha relación entre actitud y rendimiento, se realiza un análisis en alumnos de primer curso de Grado en Derecho en la materia de Fundamentos de Economía de la Empresa, considerada de bajo interés para los alumnos. Se obtienen los resultados de aplicación de metodologías cooperativas en el cambio de la satisfacción e interés de los alumnos hacia la materia mediante escalas, y se observan las ventajas e inconvenientes de su desarrollo en las aulas.
\end{abstract}

Palabras clave: actitud, clima del aula, metodología cooperativa, innovación docente.

\section{Changing Attitudes and Classroom Climate Using Cooperative Methodologies on Low Interest Subjects}

\begin{abstract}
Leaving of the narrow existent relationship between social climate and outcome, an analysis is realized in students of Fundamentals of Corporate Economics of Bachelor of Law, because of their scanty interest in the subject. After applying cooperative methodologies, it has been used scales to measure the changes of attitude and satisfaction. In addition, the advantages and disadvantages of implementing the methodologies in the classroom have been evaluated.
\end{abstract}

Keywords: attitude, climate of class, cooperative methodologies, educational innovation

\section{Referencia normalizada}

FLORES ASENJO, María Pilar (2013): "Cambio de actitud y clima del aula mediante el uso de metodología cooperativa en materias de bajo interés para el alumno". Estudios sobre el Mensaje Periodístico. Vol. 19, Núm. especial marzo, págs.: 169-175. Madrid, Servicio de Publicaciones de la Universidad Complutense.

Sumario: 1. Introducción. 2. Metodología. 3. Resultados. 4. Conclusiones. 5. Referencias bibliográficas.

\section{Introducción}

Fundamentos de Economía de la Empresa es una asignatura que forma parte del programa de Grado de Derecho y que se imparte en el primer curso académico. La actitud general observada de los alumnos hacia materias ajenas al mundo no jurídico, sobre todo si no se cuenta con preparación inicial en los años previos a la Universidad, suele ser de desinterés y mero obstáculo que hay que superar.

Como las actitudes se correlacionan con el interés por el estudio, el rendimiento, el manejo de los contenidos y el logro de los objetivos educativos, si se conocen las actitudes de los alumnos hacia el estudio, es posible fomentar y fortalecer las actitudes facilitadoras del aprendizaje, lo que conlleva una mejora global del proceso de enseñanza-aprendizaje (Schibeci y Riley, 1986). Es por esta razón por la que se optó por analizar el perfil actitudinal inicial del alumno y observar si el uso de las metodologías de aprendizaje cooperativo mejoraba o no la situación de partida. 
El aprendizaje cooperativo busca construir el conocimiento de forma conjunta entre el profesor y los alumnos, promueve la implicación activa del estudiante en el proceso e incrementa el rendimiento mediante la interacción con los compañeros. Entre las ventajas que destacan Benito y Cruz (2005) se encuentra que genera mayor entusiasmo y motivación, apoya a los alumnos "de riesgo" (alumnos de primeros cursos) y desarrolla habilidades interpersonales y de trabajo en equipo. Otros beneficios que nos interesan del aprendizaje cooperativo es que realza la satisfacción del estudiante con la experiencia de aprendizaje, reduce la ansiedad de los alumnos (Panitz, 1999) y promueven el logro cognitivo y rendimiento académico al ofrecer a los estudiantes "mayor oportunidad para discutir, para aprender unos de otros y para alentar la excelencia entre ellos" (Slavin y Cooper, 1999: 648).

Entre las distintas posibilidades que ofrece esta metodología, se aplicó la técnica Jigsaw II (Slavin, 1986). En esta técnica el profesor prepara todo el tema y luego cada miembro del grupo (de 5 alumnos) desarrolla los subtemas en "grupos de expertos". A continuación, los equipos se encargan de que todos sus miembros tengan un conocimiento completo de lo trabajado, y la evaluación se realiza por la media de las puntuaciones obtenidas por todos los miembros en la resolución de forma individual de un caso práctico sobre el tema completo. Durante todo el proceso el profesor aporta información y asesoramiento a los grupos de expertos, y supervisa el trabajo de los equipos.

Otra técnica puesta en práctica fue la de "Estructuras de Controversia" (Constructive Controver; Johnson y Johnson, 1994), en la que grupos de 4 alumnos debaten dos a dos sobre posturas diferentes de un tema para, a continuación, defender la postura contraria. El objetivo es buscar argumentaciones "a favor" y "en contra" y llegar a un consenso (que se recoge en una memoria de trabajo que sirve de autoevaluación). La evaluación individual exige al alumno el conocimiento de ambas posturas.

Para obtener una calificación actitudinal inicial de los alumnos se utilizó la Escala de Actitudes hacia el estudio de las Ciencias Básicas (E.A.C.B.), desarrollada y validada por Ortega-Ruiz et al (1992), y modificada para nuestro caso. La media de calificación obtenida fue de $3,898( \pm 0,811)$ sobre 10 , por lo que se decidió aplicar los métodos descritos y se volvió a evaluar mediante el EACB la variación de la actitud hacia la materia. También se analizó el clima del aula para ponderar cómo percibieron los alumnos el cambio en la metodología y la forma de evaluación, para seguir trabajando en su mejora. El análisis del clima del aula se realizó mediante la aplicación de una modificación de la Escala de Inventario de Ambiente de Clase en Facultades y Escuelas Universitarias (C.U.C.E.I.) de Fraser, Treagust y Dennis (1986).

\section{Metodología}

Para obtener una calificación actitudinal la EACB fue aplicada a 60 (de 64) alumnos de Fundamentos de Economía de la Empresa del Grado de Derecho (2011-12) con una edad media de 19,9 $( \pm 4,41)$.Esta escala modificada, que consta de trece ítems ( 7 positivos y 6 negativos) con 5 posibles respuestas tipo Likert, fue validada al contrastar la consistencia interna mediante el coeficiente de Cronbach (superiores a 0.7) y la validez discriminante a través de las correlaciones entre dimensiones (superiores 
a 0.6). La calificación de la actitud se obtuvo como resultado de multiplicar el valor de correlación de los datos obtenidos en la muestra y los datos esperados por la escala actitudinal multiplicado por 10 .

El análisis del clima del aula, desarrollado al final de la aplicación de las metodologías cooperativas, se realizó mediante la CUCEI modificada para el caso. Esta escala consta de 49 ítems agrupados en 7 dimensiones: personalización, implicación, cohesión entre los estudiantes, satisfacción, orientación hacia la tarea, innovación e individualización. Dado el carácter de nuestro estudio, se centró el análisis en la implicación (existencia de conductas participativas e implicación en las actividades), satisfacción (grado en que los estudiantes perciben la clase como amena e interesante) y la orientación de la tarea (grado en que perciben la clase como correctamente organizada). Estas tres dimensiones con sus 21 ítems, con 5 posibles respuestas tipo Likert, se validaron de igual forma que la escala actitudinal.

De esta forma, se sigue la propuesta de Fraser (1989) al utilizar las escalas siguiendo el proceso de evaluación inicial, retroacción o explicación de los resultados obtenidos, reflexión sobre las posibilidades de acción, intervención o puesta en práctica de las acciones negociadas y nueva evaluación con la escala para observar los cambios.

\section{Resultados}

En la encuesta EACB realizada después de aplicar la metodología cooperativa se obtuvo una calificación notablemente superior a la inicial, al pasar de $3,898( \pm 0,811)$ sobre diez a 5,405 $( \pm 0,61)$. Es decir, se aprecia una mejora sustancial del grado de interés del alumnado en la materia con respecto a la situación inicial o de partida.

El análisis de los resultados de la encuesta inicial y final muestra diferencias significativas en las medias obtenidas de todos los ítems, principalmente del ítem 3 ("Me gusta resolver casos relacionados con la Economía y Empresa"), 7 ("Las asignaturas de Economía sólo sirven para suspender y obtener malas notas") y 10 ("Las clases de Economía se me hacen aburridas y pesadas"). Aunque son menos significativas, también son relevantes las diferencias en el ítem 2 ("El estudio de la materia de Economía me resulta algo pesado porque no veo su utilidad"), 6 ("No me gustan las materias de Economía porque su estudio me parece difícil") y 13 ("Normalmente desconecto en las clases de Economía").

Los peores resultados en ambas encuestas se obtienen en los ítems 2 ("El estudio de la materia de Economía me resulta algo pesado porque no veo su utilidad"), 4 ("Me resulta desagradable estudiar la materia de Economía"), 10 ("Las clases de Economía se me hacen aburridas y pesadas") y 13 ("Normalmente desconecto en las clases de Economía"), aunque, también coincide con los ítems que mejor han evolucionado tras el uso de la metodología cooperativa. En general, los ítems negativos (que indican grado de insatisfacción o desinterés) son menos negativos y los positivos son más positivos, tan sólo se diferencian en la intensidad de los cambios. Parece, por tanto, que después del uso de las técnicas de cooperación aplicadas se ha logrado mejorar de forma general el interés por la materia, y el alumno tiene una mayor conciencia de la utilidad que la misma le reporta en su futuro profesional. 
La mayor puntuación de la escala modificada CUCEI recae sobre la dimensión "satisfacción" y "orientación de la tarea", encontrándose los mínimos en la dimensión "implicación". Aparecen como importantes inconvenientes a las metodologías aplicadas: "Organizar el trabajo en el grupo para implicar de igual forma a todos los compañeros es complicado" $(3,03 \pm 0,82)$, seguida de "Es difícil resolver los conflictos que surgen durante el desarrollo de los trabajos" $(2,96 \pm 0,88)$, siendo las principales ventajas "Participar en el proceso de aprendizaje me motiva al estudio" $(3,32 \pm 0,68)$ y "Las prácticas han aumentado mi interés por la Economía" $(3,12 \pm 0,75)$.

Las ventajas que aprecia el alumnado en la aplicación de la metodología cooperativa apoyan la decisión de su utilización en materias que parten de un bajo interés inicial. Los inconvenientes encontrados pueden ser subsanados por una profunda y eficiente planificación de las acciones por parte del profesorado, y por la constante supervisión de las aportaciones y resultados de los grupos de trabajo. También juega un papel importante el tipo de evaluación utilizado y su planificación y comunicación a los alumnos.

Las dificultades principales en el uso de las metodologías cooperativas son evitar el desequilibrio en la participación y los conflictos que puede generar, motivar el trabajo en equipo de alumnos no habituados a esta metodología, preparar la documentación adecuada a este tipo de aprendizaje y encontrar una forma correcta de evaluación de los resultados. Esto exige, por tanto, una supervisión constante del profesor, que suscite un clima adecuado de cooperación, y una eficaz programación y organización de la dinámica.

El cambio en el modelo de metodologías docentes implica un cambio en la planificación y ejecución de la docencia, pero también un cambio en la evaluación (Rué, 2008: 3). La literatura de Psicología ha destacado que la identidad social y el compromiso de los miembros del equipo con su grupo influye positivamente en el rendimiento del equipo (Haslam et al., 2006), pero se ha de poner especial cuidado en la elección del sistema de evaluación, que ha de ser comprendido correctamente por los alumnos. En dicha evaluación es recomendable que se tengan en consideración las competencias de trabajo en grupo, y no sólo el resultado individual de los alumnos, que puede ser analizado a través de las memorias de trabajo o mediante historial de correcciones si se utilizan las herramientas TIC (como por ejemplo Google Drive).

En la evaluación es aconsejable que exista una puntuación tanto del trabajo en grupo, como del logro individual del alumno como resultado de dicha colaboración. Este tipo de evaluación desincentiva el "pasotismo" o "parasitismo" de determinados componentes del grupo, que es uno de los principales inconvenientes del trabajo grupal.

\section{Conclusiones}

Son muchos los grados que incorporan materias del área de la Economía y la Empresa como parte de la formación integral para capacitar al alumno según el marco del Espacio Europeo de Educación Superior (Grados en Comunicación, Relaciones Públicas, Periodismo, Ingenierías, etc.) y, como ha podido apreciarse en este estudio, la utilización de metodologías cooperativas puede resultar una buena estrategia para au- 
mentar el interés de materias que, como la Economía en el Grado en Derecho, parten de un débil interés para el alumno.

Uno de los objetivos para los docentes que más explícitamente se subraya en el nuevo marco formativo es el de no sólo enseñar, sino también enseñar a aprender, lo que pone de relieve la necesidad de adoptar diferentes metodologías docentes que generen mayor motivación (Arquero y Jiménez, 1999). Para implementar un ambiente de aprendizaje efectivo Jonassen (1994) propone una serie de características generales de aprendizaje como enfatizar la construcción del conocimiento en vez de la reproducción del mismo, fomentar la reflexión basada en la experiencia, y apoyar la construcción colaborativa del conocimiento a través de la negociación social y no la competencia.

Al utilizar la metodología cooperativa se busca que los alumnos obtengan la capacidad de realizar hallazgos de forma autónoma y grupal, la adquisición de una mayor autoestima, una mayor conciencia de su capacidad social y una mejoría de la actitud proactiva (Peña y Parra, 2012). Los estudiantes toman conciencia de la importancia de una buena planificación, de la diversidad de formas en las que se puede llevar a cabo y de las consecuencias que pueden tener en el óptimo desarrollo del trabajo en equipo (Flores, 2011). La colaboración fomenta que los estudiantes se sientan parte de una comunidad de aprendizaje, lo que favorece la construcción de conocimiento compartido y crea un espíritu comunitario (Garrison y Anderson, 2005; TU, 2004). Las relaciones que se producen en el aula afectan al grado de comunicación favoreciendo un clima social adecuado para despertar el interés por la materia.

Para que este tipo de experiencias tenga éxito es recomendable que el profesor exponga con claridad las tareas a realizar, los objetivos que se persiguen y que se produzca un continuo feedback sobre su consecución (Alonso, 2007). El docente deberá estar presente en cada uno de los pasos del alumno como orientador y guía de la experiencia de aprendizaje, lo que supone haber realizado previamente una correcta programación de la misma, pero además, la evaluación es una herramienta fundamental para una correcta comunicación- En ella es recomendable que se tengan en consideración las competencias de trabajo en grupo, y no sólo el resultado individual de los alumnos, ya que este tipo de evaluación desincentiva el "pasotismo" de determinados componentes del grupo (que es uno de los principales inconvenientes del trabajo en grupo).

Si se quiere emplear nuevas metodologías educativas, los primeros que deberán formarse son los propios profesores. El papel del formador requiere una buena documentación, liderazgo de grupo, ser proactivo y empático, para minimizar los aspectos negativos observados en su puesta en práctica (Parra y Peña, 2012).

\section{Referencias bibliográficas}

ALONSO, Pilar (2007): "Evaluación formativa y su repercusión en el clima del aula" Revista de Investigación Educativa 25(2); pp. 389-402.

ARQUERO, José L. y Sergio M. JIMÉNEZ (1999): “Influencia del estudio de casos en la mejora del aprendizaje, adquisición de capacidades no técnicas y motivación 
en análisis contable". Revista de Enseñanza Universitaria Extraordinario $\mathrm{n}^{\circ} 1$; pp. 225-242.

BENITO, Águeda y CRUZ, Ana (2005): Nuevas claves para la docencia universitaria. Madrid, Narcea.

FLORES, Pilar (2011): "Una experiencia de trabajo colaborativo: Uso de Office Live Workspace en la elaboración de un trabajo de investigación en equipo" En PEÑA, B. (coord.): Nuevas fórmulas docentes en el EEES. Madrid, Visión Libros.

FRASER, Barry J. (1989): "Twenty years of classroom climate work: progress and prospect". Journal of Curriculum Studies 21 (4); pp. 307-327.

FRASER, Barry; TREAGUST, David y DENNIS, Norman (1986): "Development of an instrument for assessing classroom psychosocial environment at universities and colleges" Studies in Higher Education 11 (1); pp. 43-54.

GARRISON, D. Randy y ANDERSON, Terry (2005): El e-learning en el siglo XXI. Barcelona. Octaedro.

HASLAM, S. Alexander; RYAN, Michelle K.; POSTMES, Tom; SPEARS, Rusell; JETTEN, Jolanda; y WEBLEY, Paul (2006): "Sticking to our guns: Social identity as a basis for the maintenance of commitment to faltering organizational projects". Journal of Organizational Behaviour, 27; pp. 607-628.

JOHNSON, David W. y JOHNSON, Roger T. (1994): Learning together and alone: Cooperative, competitive, and individualistic learning ( $4^{\mathrm{a}}$ edición). Needham Heights. Ally and Bacon.

JONASSEN, David H. (1994): What might constructivism mean in education? Penn State Education Summit: Issues on the Forefront. University Park, PA.

ORTEGA-RUÍZ, Pedro; SAURA, José P.; MÍNGUEZ, Ramón; GARCÍA DE LAS BAYONAS, Antonio; y MARTÍNEZ, Daniel (1992): "Diseño y aplicación de una escala de actitudes hacia el estudio de las Ciencias Experimentales". Enseñanza de las Ciencias 10(3); pp. 295-303.

PANITZ, Ted (1999): The case for student centred instruction via collaborative learning paradigms. En línea: http://www.capecod.net/ TPanitz/Tedspage (fecha de consulta: 4 de junio de 2012).

PARRA, M. Concepción y PEÑA, Beatriz (2012): "El aprendizaje cooperativo mediante actividades participativas" Anales de la Universidad Metropolitana, 12(2); pp. 15-42.

PEÑA, Beatriz y PARRA, M. Concepción (2012): “Aprendizaje cooperativo en observación sistemática mediante el visionado de Films". Revista de Comunicación Vivat Academia XIV (número especial); pp. 701-712.

RUÉ, Joan (2008): "Formar en competencias en la universidad: entre la relevancia y la banalidad". Red U. Revista de Docencia Universitaria, número monográfico 1. Disponible en: http://www.redu.m.es/Red_U/m1 (fecha de consulta: 6 de Abril de 2011). 
SCHIBECI, Renato A. y RILEY, J. Patrick (1986): “Influence of students’ background and perceptions on science attitudes and achievement" Journal of Research in Science Teaching, 23(3); pp. 177-189.

SLAVIN, Robert E. y COOPER, Robert (1999): "Improving intergroup relations: Lessons learned from cooperative learning programs" Journal of Social Issues 55; pp. 647-663.

TU, Chih-Hsiung (2004): Online collaborative learning communities: Twenty-one designs to building an online collaborative learning community. Westport. CT: Libraries Unlimited.

\section{María Pilar FLORES ASENJO}

Universidad Católica San Antonio de Murcia (UCAM)

Facultad de Ciencias Jurídicas y de la Empresa

Pflores@ucam.edu

Profesora Doctora en Administración y Dirección de Empresas de la UCAM

Coordinadora departamental de Relaciones Internacionales de la UCAM y miembro del cuerpo de tutores

Miembro del Grupo de Investigación “Organización de empresas y marketing” 